Conferencias, seminarios y trabajos de Matemática

ISSN: 1515.4904

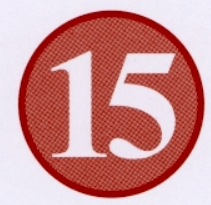

Workshop on

Mathematical

Modelling of Energy

and Mass Transfer

Processes, and

Applications

Domingo A. Tarzia

Rodolfo H. Mascheroni (Eds.) 


\title{
CONSIDERACIONES SOBRE EL BUEN PLANTEO DE UN MODELO DE FRONTERA LIBRE - MOVIL DESCRIPTIVO DE UN PROCESO DE FREÍDO POR INMERSIÓN
}

\author{
Luis T. VILLA \\ Univ. Nac. de Salta, Salta, Argentina \\ E-mail: villal@unsa.edu.ar
}

\begin{abstract}
Resumen
El objetivo del presente trabajo consiste en abordar un análisis concerniente a la cuestión básica del buen planteo de la solución de un problema de frontera libre-móvil, unidimensional a dos fases para la ecuación del calor y difusión de materia, emergente del modelado de la etapa principal de un proceso de freído de papa natural por inmersión en aceite comestible caliente. Se provee un resultado para la unicidad de la solución del modelo analizado. También se obtiene un resultado de dependencia monótona del avance de la frontera libre con la temperatura del baño de aceite, y con la temperatura inicial de la porción a freir.
\end{abstract}

\begin{abstract}
The goal of the present work concern to the basic question of well-posedness of the solution of a one dimensional two phase initial free-moving boundary value problem for the heat-diffusion equation. Such problem arise from the corresponding mathematical modeling of the so called principal stage (bubbling stage) during a immersion frying process, applied to natural potato. A result regarding the uniqueness of the solution is provided. Also, a result on monotone dependence for the progress of the free boundary with the oil bath temperature, and with the initial temperature of the potato sample are consigned.
\end{abstract}

\section{Nomenclatura}

$x, t$ : Variables independientes, espacial y tiempo respectivamente (m), (seg).

$A$ : Constante positiva, área superficial principal de la porción de papa $\left(\mathrm{m}^{2}\right)$.

$C=C(x, t):$ Perfil de concentración de agua líquida $\left(\mathrm{kg} / \mathrm{m}_{t}^{3}\right)$.

$\bar{C}=\bar{C}(x, t)$ : Función concentración adimensional de agua líquida definida por (22).

$F(t), f(t)$ : Funciones del tiempo t, definidas por (20) y (21) respectivamente.

$L, \ell$ : Semiespesor de la porción prismática de papa (m) y parámetro definido por (18), respectivamente.

$m, p_{o}, p_{1}, q, r$ : Parámetros definidos por (18), (15), (16) y (25) respectivamente.

$S(t)$ : Función frontera libre o coordenada móvil que ubica la interfase Corteza-Corazón en la región sólido papa $(\mathrm{m})$.

$t_{2}$ : Tiempo conllevado por la etapa del freído previa a la etapa principal (seg).

$T=T(x, t), U=U(x, t)$ : Perfiles de temperatura en las zonas Corazón y Corteza de la papa respectivamente $\left({ }^{\circ} \mathrm{C}\right)$. 
$\bar{T}=\bar{T}(x, t), \bar{U}=\bar{U}(x, t)$ : Perfiles de temperatura en las zonas Corazón y Corteza, definidas por (22) $\left({ }^{\circ} \mathrm{C}\right)$.

$T_{b}, T_{e}, T_{0}$ : Temperaturas de: baño de aceite, ebullición del agua, inicial de la porción de papa, respectivamente $\left({ }^{\circ} \mathrm{C}\right)$.

$G, V$ : Funciones definidas por (42) y (43) respectivamente.

$Z$ : Variable espacial independiente adimensional definida por (22).

Subíndices:

$t$ : Total

Propiedades del sólido papa o Termodinámicas:

$C_{c}, C_{s}, C_{v}$ : Calores específicos de las zonas Corteza, Corazón y vapor de agua a $100^{\circ} \mathrm{C}$, respectivamente $\left(\mathrm{J} / \mathrm{kg}^{\mathrm{o}} \mathrm{C}\right)$.

$D$ : Difusividad efectiva del agua líquida $\left(\mathrm{m}^{2} / \mathrm{seg}\right)$.

$h$ : Coeficiente global de transferencia de calor $\left(\mathrm{W} / \mathrm{m}^{2 \mathrm{o}} \mathrm{C}\right)$.

$h_{c}, h_{s}$ : Entalpías de las zonas Corteza y Corazón respectivamente $(\mathrm{J} / \mathrm{kg})$.

$h_{a}, H_{v}$ : Entalpías del agua y vapor a $100^{\circ} \mathrm{C}$, respectivamente $(\mathrm{J} / \mathrm{kg})$.

$\Delta H_{v}$ : Calor latente de vaporización del agua $(\mathrm{J} / \mathrm{mol})$.

$k_{c}, k_{s}$ : Conductividades térmicas de las zonas Corteza y Corazón respectivamente $\left(\mathrm{W} / \mathrm{m}^{\circ} \mathrm{C}\right)$.

$K_{v}$ : Permeabilidad (Conductividad hidráulica) $\left(m^{2}\right)$.

$V_{a}, V_{v}$ : Volúmenes molares específicos del agua y vapor a $100^{\circ} \mathrm{C}\left(\mathrm{m}^{3} / \mathrm{mol}\right)$.

\section{Símbolos Griegos}

$\mathcal{E}_{c}, \mathcal{E}_{s}, \mathcal{E}_{v}$ : Fracción de volumen $\left(\mathrm{m}^{3} / \mathrm{m}_{t}^{3}\right)$ de las zonas Corteza, Corazón y vapor de agua respectivamente.

$\rho_{c}, \rho_{s}, \rho_{v}$ : Densidades $\left(\mathrm{kg} / \mathrm{m}^{3}\right)$ de las zonas Corteza, Corazón y vapor de agua a $100^{\circ} \mathrm{C}$, respectivamente.

$\mu_{v}$ : Viscosidad del vapor de agua a $100^{\circ} \mathrm{C}$.

$\alpha, \beta, \gamma, \delta, \mu, \lambda, \nu, \Lambda$ : Parámetros definidos por (23) у (24).

$\omega=\omega(t), \Omega=\Omega(t)$ : Funciones definidas por (37) y (38) respectivamente.

$\Omega_{1}, \Omega_{2}$ : Regiones definidas por (41) y (42) respectivamente.

$\tau$ : Variable tiempo desplazada definida por (22).

\section{Introducción:}

En el presente nos abocaremos a realizar un análisis básico desde el punto de vista matemático, cual es el atinente a cuestiones relativas al buen planteo (comenzando por la unicidad de solución) de un problema de valor inicial y frontera libre-móvil, como modelo matemático descriptivo de un proceso de freído de papa natural por inmersión en aceite comestible caliente.

Tal proceso, puede caracterizarse fenomenológicamente como ocurriendo según tres etapas consecutivas temporalmente, a saber: 
Etapa 0 (de precalentamiento del sólido).

Etapa 1 (Vaporización de humedad "libre” o "agua capilar”).

Etapa 2 (la denominamos etapa principal en el presente. También se denomina de burbujeo. Es la más larga del proceso) (Para detalles ver [1], [2]).

En el presente, específicamente centramos el análisis en la etapa principal.

En las figuras 1, 2 y 3 se ilustran el dominio sólido (papa en porción prismática) considerado, las dos zonas caracterizantes del sólido durante la evolución dinámica de la etapa y los correspondientes perfiles de temperatura y humedad, de ocurrencia esperada en el sólido, respectivamente.
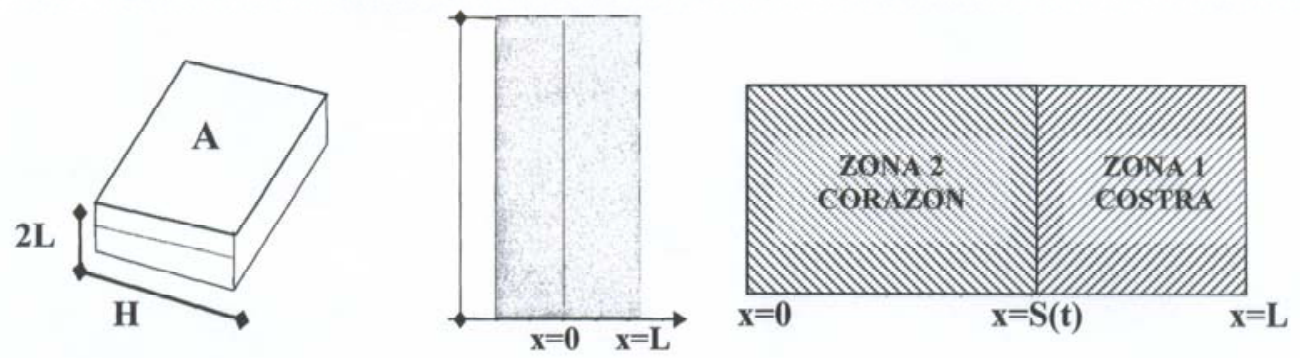

Fig 1: Esquema de la geometría de muestra de papa usada

Fig 2

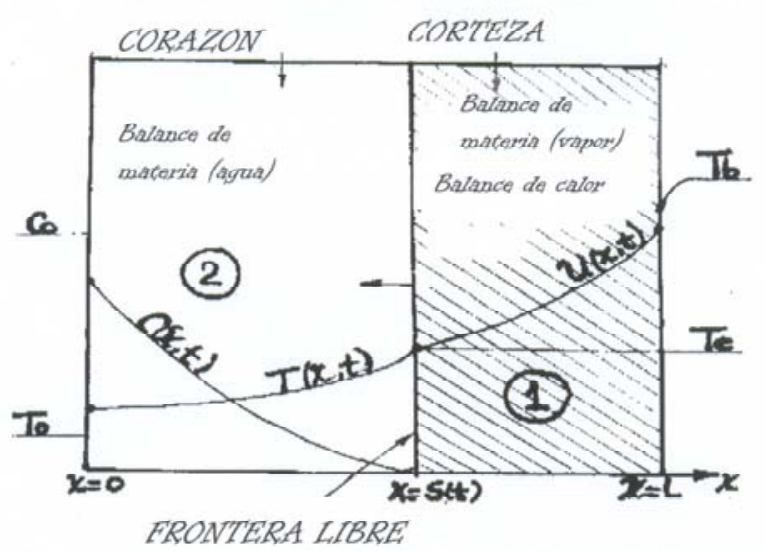

Fig 3: Diagrama esquemático de perfiles de temperatura y concentración de agua en la papa durante la Etapa principal

Como puede apreciarse, en el modelo asumido la vaporización del agua contenida en la papa se postula ocurriendo esencialmente sobre un frente o superficie móvil cuya coordenada viene justamente proporcionada por la función frontera libre $S=S(t)$, esperándose ser una función monótona decreciente desde su valor inicial $S(0)=L$ coincidente con la superficie de contacto papa- baño de aceite, moviéndose hacia el centro de simetría de la porción sólida al transcurrir el proceso.

Entonces, durante la etapa en análisis, el dominio sólido viene caracterizado por las dos zonas esquemáticamente ilustradas en la Fig. 2:

Zona 1: Corteza ó Costra, $S(t)<x<L$

Zona 2: Corazón, $0<x<S(t)$

Con la ocurrencia de los siguientes fenómenos de transporte de calor y materia:

Zona Corazón: Conducción de calor y transporte de agua líquida por difusión molecular, para vaporizarse sobre el frente $\mathrm{x}=\mathrm{S}(\mathrm{t})$.

Zona Corteza: Conducción de calor y transporte de vapor de agua por gradiente de presión (se asume un flujo sin resistencia prácticamente a través de un medio poroso). 
En la Fig. 4 se ilustra esquemáticamente los dominios y condiciones de contorno correspondientes a cada función incógnita ocurrentes en el proceso

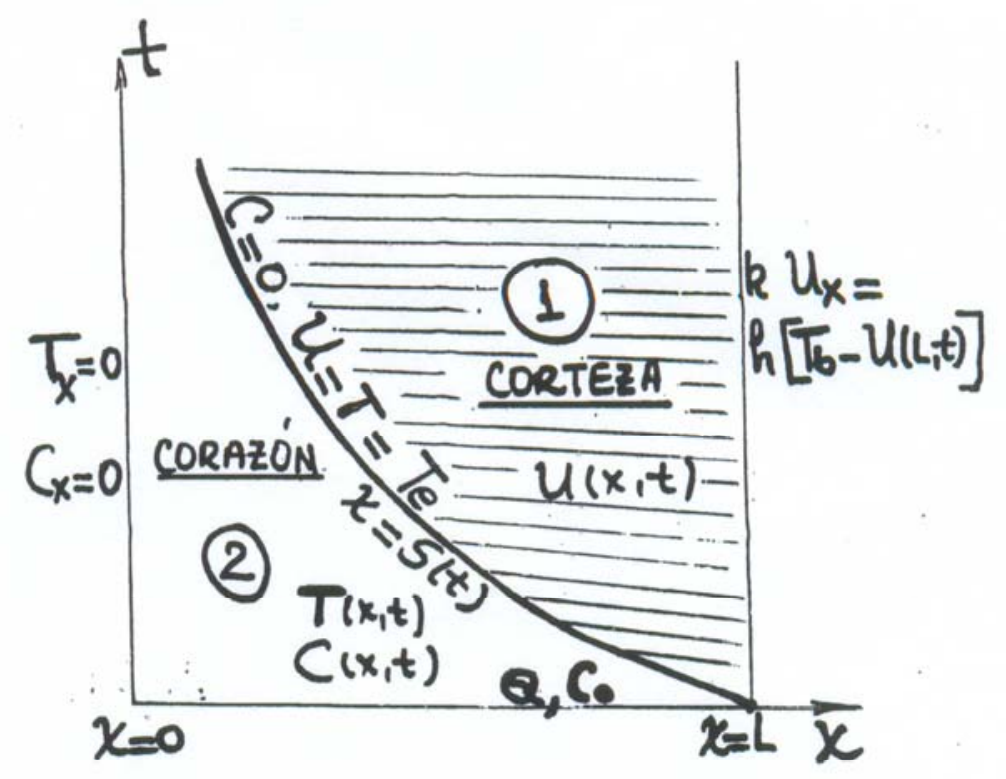

Fig 4: Esquema de dominios y condiciones de contorno correspondientes a cada función incógnita

\section{Modelo Matemático Descriptivo}

Bajo el supuesto del cumplimiento de un cierto conjunto de hipótesis y restricciones oportunamente contempladas y respetando la notación consignada en la nomenclatura referente a variables, parámetros y funciones, emerge el siguiente modelo matemático descriptivo de la evolución del proceso de freído durante la etapa considerada:

$$
\begin{aligned}
& \varepsilon_{s} \rho_{s} C_{s} \frac{\partial T}{\partial t}=k_{s} \frac{\partial^{2} T}{\partial x^{2}}, \quad 0<x<S(t), \quad t>t_{2}, \\
& \frac{\partial T}{\partial x}(0, t)=0, \quad t>t_{2}, \\
& T(S(t), t)=T_{e} \quad, \quad t>t_{2}, \\
& T=Q(x), \quad 0 \leq x \leq L, \quad t=t_{2}, \\
& Q(x)=1.52 \exp (0.8) x^{3}-9.13 \exp (0.5) x^{2}+2.05 \exp (0.3) x+20, \\
& \left(\mathcal{E}_{v} \rho_{v} C_{v}+\mathcal{E}_{c} \rho_{c} C_{c}\right) \frac{\partial U}{\partial t}=k_{c} \frac{\partial^{2} U}{\partial x^{2}}, \quad S(t)<x<L, \quad t>t_{2}, \\
& k_{c} \frac{\partial U}{\partial x}(L, t)=h\left[T_{b}-U(L, t)\right], \quad t>t_{2}, \\
& U(S(t), t)=T_{e}, \quad t>t_{2}, \\
& \frac{\partial C}{\partial t}=D \frac{\partial^{2} C}{\partial x^{2}}, \quad 0<x<S(t),
\end{aligned}
$$




$$
\begin{aligned}
& \frac{\partial C}{\partial x}(0, t)=0, \quad t>t_{2}, \\
& C(S(t), t)=0, \quad t>t_{2}, \quad t=t_{2}, \\
& C=C_{0}, \quad 0 \leq x \leq L, \quad \\
& S\left(t_{2}\right)=L .
\end{aligned}
$$

\section{Balance de Energía sobre la Frontera Libre $S(t)$}

$$
p_{0} \frac{d S}{\partial t}=p_{1} \frac{\partial U}{\partial X}(S(t), t)+k_{s} \frac{\partial T}{\partial x}(S(t), t), \quad \mathrm{t}>\mathrm{t}_{2}
$$

donde los parámetros $p_{0}$ y $p_{1}$ vienen dados como

$$
\begin{aligned}
& p_{0}=\mathcal{E}_{s} \rho_{s}\left(h_{c}-h_{s}\right)+\mathcal{E}_{v} \rho_{v}\left(H_{v}-\rho_{d}\right), \\
& p_{1}=\frac{\left(H_{v}-h_{a}\right) \rho_{v} K_{v} \Delta H_{v}}{T_{e} \mu_{v}\left(V_{v}-V_{a}\right)}-k_{c} .
\end{aligned}
$$

Balance de Materia sobre la Frontera Libre $S(t)$

$$
m \frac{d S}{d t}=D \frac{\partial C}{\partial x}(S(t), t)-\ell \frac{\partial U}{\partial x}(S(t), t) \quad, t>t_{2},
$$

con los parámetros $m$ y $\ell$ dados como

$$
m=\varepsilon_{a} \rho_{a}-\varepsilon_{v} \rho_{v} \quad, \quad \ell=\frac{\rho_{v} K_{v}}{\mu_{v} T_{e}} \frac{\Delta H_{v}}{\left(V_{v}-V_{a}\right)} .
$$

\section{Observación 1}

En adelante la condición de contorno dada por la ecuación (7), para el perfil de temperatura U, se reemplazará por la siguiente:

$$
k_{c} \frac{\partial U}{\partial x}(L, t)=F(t), \quad t>t_{2}
$$

con $F=F(t)$ dada como:

$$
F(t)=h\left[T_{b}-f(t)\right], \quad t>t_{2},
$$

donde

$$
f(t)=1 \exp (-10) t^{4}-3 \exp (-0.7) t^{3}+0.0003 t^{2}-0.0037 t+108.82
$$

\section{Observación 2}

Se debe tener presente en adelante que la condición de contorno sustitutiva consignada por la ecuación (19) proviene de datos experimentales de la historia térmica del proceso medida en un punto muy cercano a la superficie externa de contacto papa - aceite. En efecto, $f=f(t)$ dado por la ecuación (21) es una función de ajuste de los citados datos . 


\section{Observación 3}

Con vista a efectuar el tratamiento del Modelo descriptivo consignado por la ecuaciones (1) a (21) explicitadas precedentemente, conviene trabajar sobre una versión equivalente a la dada por tales ecuaciones. A tal efecto, se introducen las siguientes transformaciones de variables y funciones a saber:

$$
\begin{aligned}
& Z=1-\frac{x}{L}, \quad \tau=t-t_{2}, \quad \sigma(t)=\frac{L-S(t)}{L}, \\
& \bar{C}(x, t)=\frac{C(x, t)}{C_{0}}, \quad \bar{T}(x, t)=T_{e}-T(x, t), \\
& \bar{U}(x, t)=U(x, t)-T_{e}
\end{aligned}
$$

y además los siguientes parámetros nuevos, definidos en términos de los básicos:

$$
\begin{aligned}
& \alpha=\frac{k_{s}}{\rho_{s} C_{s} \varepsilon_{s} L^{2}}, \quad \beta=\frac{k_{c}}{L^{2}\left(\varepsilon_{v} \rho_{v} C_{v}+\rho_{c} \varepsilon_{c} C_{c}\right)}, \quad \Lambda=\frac{D}{L^{2}}, \\
& \lambda=\frac{k_{s}}{p_{0} L^{2}}, \quad v=\frac{p_{1}}{p_{0} L^{2}}, \quad \gamma=\frac{v}{\beta}, \delta=\frac{\lambda}{\alpha}, \mu=\frac{v L}{k_{c}}, \\
& q=\frac{D C_{0}}{m L^{2}}, \quad r=\frac{\ell}{L^{2} m} .
\end{aligned}
$$

Entonces, teniendo presente la Observación 1, las transformaciones dadas por (22) y (23) a (25), trabajando sobre las ecuaciones (1) a (21) se obtiene la siguiente versión equivalente, donde en pro de facilitar la escritura y no complicarse con tanta nueva simbología, las funciones $\bar{U}, \bar{T}, \bar{C}$ se volvieron a denotar con $\mathrm{U}, \mathrm{T}, \mathrm{C}$ y a la variable tiempo $\tau$ como $\mathrm{t}$

$$
\begin{aligned}
& (I)\left\{\begin{array}{l}
\frac{\partial U}{\partial t}=\beta \frac{\partial^{2} U}{\partial Z^{2}}, \quad 0<Z<\sigma(t), \quad t>0, \\
-\frac{k_{c}}{L} \frac{\partial U}{\partial Z}=F(t), \quad Z=0, \quad t>0, \\
U=0, \quad Z=\sigma(t), \quad t>0,
\end{array}\right. \\
& (I I)\left\{\begin{array}{l}
\frac{\partial T}{\partial t}=\alpha \frac{\partial^{2} T}{\partial Z^{2}}, \quad \sigma(t)<Z<1, \quad t>0, \\
\begin{array}{l}
\frac{\partial T}{\partial Z}=0, \\
T=0
\end{array} \quad Z=\sigma(t), \quad t>0, \\
T=W(Z)=T_{e}-Q(L-L Z), \quad 0 \leq Z \leq 1, \quad t=0,
\end{array}\right. \\
& (I I I)\left\{\begin{aligned}
\frac{d \sigma}{d t} & =v \frac{\partial U}{\partial Z}(\sigma(t), t)-\lambda \frac{\partial T}{\partial Z}(\sigma(t), t), \quad t>0, \\
\sigma(0) & =0
\end{aligned}\right.
\end{aligned}
$$




$$
\begin{aligned}
& (I V)\left\{\begin{array}{l}
\frac{\partial C}{\partial t}=\Lambda \frac{\partial^{2} C}{\partial Z^{2}}, \quad \sigma(t)<Z<1, \quad t>0, \\
\frac{\partial C}{\partial Z}=0, \quad Z=1, \quad t>0, \\
C=0, \quad Z=\sigma(t), \quad t>0, \\
C=1, \quad 0 \leq Z \leq 1, \quad t=0,
\end{array}\right. \\
& (V)\left\{\begin{array}{l}
\frac{d \sigma}{d t}=q \frac{\partial C}{\partial Z}(\sigma(t), t)-r \frac{\partial U}{\partial Z}(\sigma(t), t),
\end{array}\right.
\end{aligned}
$$

\section{Observación 4}

Desde el punto de vista conceptual, es importante puntualizar que los sistemas agrupados como (I), (II) y (III), partes del modelo descriptivo (26) a (38), constituyen un problema de frontera libre unidimensional a dos fases. En tanto que, los sistemas (IV) y (V) relacionados con (I), (II), (III) a través de la función $\sigma=\sigma(t)$, constituyen un problema de frontera móvil para la función incógnita $C=C(x, t)$.

El flujo de información es el siguiente:

- $\quad$ Se resuelve (I), (II), (III) para las incógnitas U, T, $\sigma$.

- $\quad$ Se resuelve (IV) usando la $\sigma$ del paso precedente.

- De (V) se calcula $\frac{\partial C}{\partial Z}(\sigma(t), t)$

Unicidad de la solución del Submodelo de Frontera Libre (I), (II), (III).

Supongamos que fuera posible la ocurrencia de dos soluciones distintas $\sigma_{1}, T_{1}, U_{1}$ y $\sigma_{2}, T_{2}, U_{2}$ para el problema de frontera libre consignado por (I), (II), (III). Por consiguiente, en virtud de las ecs. (26) a (33) ((I), (II), (III)), se deberá verificar

$$
\begin{aligned}
& (I)^{\prime}\left\{\begin{array}{l}
\frac{\partial U_{1}}{\partial t}=\beta \frac{\partial^{2} U_{1}}{\partial Z^{2}}, 0<Z<\sigma_{1}(t), \quad t>0, \\
-\frac{k c}{L} \frac{\partial U_{1}}{\partial Z}=F(t), \quad Z=0, \\
U_{1}=0, \quad Z=\sigma_{1}(t), \quad t>0,
\end{array}\right. \\
& (I I)^{\prime}\left\{\begin{array}{l}
\frac{\partial T_{1}}{\partial Z}=\alpha \frac{\partial^{2} T_{1}}{\partial Z^{2}}, \quad \sigma_{1}(t)<Z<1, \quad t>0, \\
\frac{\partial T_{1}}{\partial Z}=0, \quad Z=1, \quad t>0, \\
T_{1}=0, \quad Z=\sigma_{1}(t), \quad t>0, \\
T_{1}=W(z), \quad 0 \leq Z \leq 1, \quad t=0,
\end{array}\right.
\end{aligned}
$$




$$
\begin{aligned}
& (I I I)^{\prime}\left\{\begin{aligned}
\frac{d \sigma_{1}}{d t} & =v \frac{\partial U_{1}}{\partial Z}\left(\sigma_{1}(t), t\right)-\lambda \frac{\partial T_{1}}{\partial Z}\left(\sigma_{1}(t), t\right) \\
\sigma_{1}(0) & =0
\end{aligned}\right. \\
& (I)^{\prime \prime}\left\{\begin{array}{l}
\frac{\partial U_{2}}{\partial t}=\beta \frac{\partial^{2} U_{2}}{\partial Z^{2}}, \quad 0<Z<\sigma_{2}(t), \quad t>0, \\
-\frac{k c}{L} \frac{\partial U_{2}}{\partial Z}=F(t), \quad Z=0, \quad t>0, \quad t>0, \\
U_{2}=0, \quad Z=\sigma_{2}(t), \quad t
\end{array}\right. \\
& (I I)^{\prime \prime} \begin{cases}\frac{\partial T_{2}}{\partial Z}=\alpha \frac{\partial^{2} T_{2}}{\partial Z^{2}}, \quad \sigma_{2}(t)<Z<1, & t>0, \\
\frac{\partial T_{2}}{\partial Z}=0, & t>0, \\
T_{2}=0 \quad Z=\sigma_{2}(t), & t>0, \\
T_{2}=W(Z), \quad 0 \leq Z \leq 1, & t=0,\end{cases} \\
& (I I I)^{\prime \prime}\left\{\begin{array}{l}
\frac{d \sigma_{2}}{d t}=v \frac{\partial U_{2}}{\partial Z}\left(\sigma_{2}(t)\right)-\lambda \frac{\partial T_{2}}{\partial Z}\left(\sigma_{2}(t), t\right) \\
\sigma_{2}(0)=0
\end{array}\right.
\end{aligned}
$$

Definimos ahora a las funciones $\omega=\omega(t), \Omega=\Omega(t)$ y a las regiones $\Omega_{1}, \Omega_{2}$ como:

$$
\begin{aligned}
& \omega(t)=\operatorname{Min}\left\{\sigma_{1}(t), \sigma_{2}(t)\right\}, \\
& \Omega(t)=\operatorname{Max}\left\{\sigma_{1}(t), \sigma_{2}(t)\right\}, \\
& \Omega_{1} \equiv\{(Z, t) / 0<Z<\omega(t), t>0\}, \\
& \Omega_{2} \equiv\{(Z, t) / \Omega(t)<Z<1, t>0\},
\end{aligned}
$$

Para tomar una de las alternativas posibles, supóngase que

$$
\sigma_{1}(t)>\sigma_{2}(t), \quad t>0 .
$$

Entonces, se pueden formular los siguientes problemas auxiliares emergentes de (I)' a (II)' y (I)' ' a (II)', teniendo presente (37) a (41): 
Villa, MAT - Serie A, Proceso de freido por inmersión, 15 (2008), 25-36

$$
\begin{aligned}
& (a) \begin{cases}\frac{\partial U_{1}}{\partial t}=\beta \frac{\partial^{2} U_{1}}{\partial Z^{2}},(Z, t) \in \Omega_{1} \equiv\left\{(Z, t) / 0<Z<\sigma_{2}(t), \mathrm{t}>0\right\} \\
-\frac{k_{c}}{L} \frac{\partial U_{1}}{\partial Z}=F(t), \mathrm{Z}=0, & \mathrm{t}>0, \\
U_{1}\left(\sigma_{2}(t), t\right)>0, & \mathrm{t}>0,\end{cases} \\
& \left\{\begin{array}{l}
\frac{\partial U_{2}}{\partial t}=\beta \frac{\partial^{2} U}{\partial Z^{2}},(\mathrm{Z}, \mathrm{t}) \in \Omega_{1} \\
-\frac{k_{c}}{L} \frac{\partial U_{2}}{\partial Z}=F(t), \mathrm{Z}=0, \quad \mathrm{t}>0 \\
U_{2}\left(\sigma_{2}(t), t\right)=0, \quad \mathrm{t}>0
\end{array}\right. \\
& \begin{array}{l}
(c)\left\{\begin{array}{l}
\frac{\partial T_{1}}{\partial t}=\alpha \frac{\partial T_{1}}{\partial Z^{2}},(Z, t) \in \Omega_{2} \equiv\left\{(Z, t) / \sigma_{1}(t)<Z<1, \mathrm{t}>0\right\} \\
\frac{\partial T_{1}}{\partial Z}=0, \mathrm{Z}=1, \quad \mathrm{t}>0, \\
T_{1}\left(\sigma_{1}(t), t\right)=0, \quad \mathrm{t}>0, \\
T_{1}(Z, 0)=W(Z), \quad 0 \leq \mathrm{Z} \leq 1, \quad \mathrm{t}=0, \\
\frac{\partial T_{2}}{\partial t}=\alpha \frac{\partial T_{2}}{\partial Z^{2}},(Z, t) \in \Omega_{2} \\
\frac{\partial T_{2}}{\partial Z}=0, \mathrm{Z}=1, \quad \mathrm{t}>0, \\
T_{2}\left(\sigma_{1}(t), t\right)>0, \quad \mathrm{t}>0, \\
T_{2}(Z, 0)=W(Z), \quad 0 \leq \mathrm{Z} \leq 1
\end{array}\right.
\end{array}
\end{aligned}
$$

Definimos ahora las funciones $G=G(z, t)$ y $V=V(z, t)$ como:

$$
\begin{aligned}
& G(z, t)=U_{1}(z, t)-U_{2}(z, t), \quad(z, t) \in \Omega_{1} \\
& V(z, t)=T_{2}(z, t)-T_{1}(z, t), \quad(z, t) \in \Omega_{2}
\end{aligned}
$$

que resultan satisfacer los siguientes problemas: 


$$
\begin{gathered}
(e)\left\{\begin{array}{l}
\frac{\partial G}{\partial t}=\beta \frac{\partial^{2} G}{\partial Z^{2}}, \quad(\mathrm{Z}, \mathrm{t}) \in \Omega_{1} \\
\frac{\partial G}{\partial Z}=0, \quad \mathrm{Z}=0, \quad t>0, \\
\mathrm{G}\left(\sigma_{2}(\mathrm{t}), \mathrm{t}\right)>0 \quad \mathrm{t}>0,
\end{array}\right. \\
(f)\left\{\begin{array}{l}
\frac{\partial V}{\partial t}=\alpha \frac{\partial^{2} V}{\partial Z^{2}}, \quad(\mathrm{Z}, \mathrm{t}) \in \Omega_{2} \\
\frac{\partial V}{\partial Z}=0, \quad \mathrm{Z}=1, \quad t>0, \\
V\left(\sigma_{1}(t), t\right)>0, \quad \mathrm{t}>0, \\
\mathrm{~V}(\mathrm{Z}, 0)=0, \quad 0 \leq \mathrm{Z} \leq 1
\end{array}\right.
\end{gathered}
$$

Teniendo presente (7), (20) y (22), en virtud del Principio del máximo aplicado a los problemas (e) y (f) se infiere la siguiente propiedad de comparación para las funciones $U_{1}, U_{2}, T_{1}, T_{2}$ :

$$
\begin{aligned}
& U_{1}(Z, t)>U_{2}(Z, t), \forall(Z, t) \in \Omega_{1}, \\
& T_{2}(Z, t)>T_{1}(Z, t), \forall(Z, t) \in \Omega_{2} .
\end{aligned}
$$

En la Fig. 5 se ilustra esquemáticamente los comportamientos emergentes para las citadas funciones.

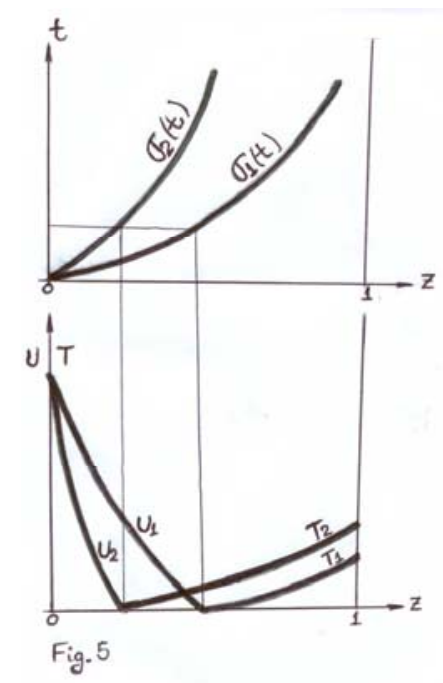

Formulación Integral a partir de (I), (II), (III) para la Frontera Libre $\sigma(t)$.

Integrando en los respectivos dominios las ecuaciones diferenciales de (I) y (II), se obtienen expresiones para $\int_{0}^{t} \frac{\partial U}{\partial Z}(\sigma(\eta), \eta) d \eta, \int_{0}^{t} \frac{\partial T}{\partial Z}(\sigma(\eta), \eta) d \eta$, lo que usado en (III) permite obtener la siguiente expresión para $\sigma(t)$ : 


$$
\sigma(t)=\gamma \int_{0}^{\sigma(t)} U(z, t) d z+\delta \int_{\sigma(t)}^{1} T(z, t) d z+\mu \int_{0}^{t} F(\eta) d \eta-\delta \int_{0}^{1} W(z) d z
$$

Teniendo presente (41), a partir de (46) se puede escribir:

$$
\begin{aligned}
& \sigma_{1}(t)-\sigma_{2}(t)=\gamma\left[\int_{0}^{\sigma_{1}(t)} U_{1}(Z, t) d Z-\int_{0}^{\sigma_{2}(t)} U_{2}(Z, t) d Z\right]+ \\
& \delta\left[\int_{\sigma_{1}(t)}^{1} T_{1}(Z, t) d Z-\int_{\sigma_{2}(t)}^{1} T_{2}(Z, t) d Z\right],
\end{aligned}
$$

donde cabe consignar que los parámetros $\gamma$ y $\delta$ verifican

$$
\gamma<0, \delta>0
$$

Entonces, teniendo presente lo establecido por las desigualdades (44), (45) y (48), de (47) se sigue que el segundo miembro de tal igualdad resulta negativo, debiendo ser el primer miembro positivo por lo supuesto explicitado por (41).

Obviamente, a la misma conclusión se arribará, si se hubiese supuesto la desigualdad contraria a la dada por (41), es decir $\sigma_{1}(\mathrm{t})<\sigma_{2}(\mathrm{t})$.

Se concluye entonces de que no pueden existir dos soluciones distintas (ternas distintas: $\left.\left(\sigma_{1}, T_{1}, U_{1}\right),\left(\sigma_{2}, T_{2}, U_{2}\right)\right)$ correspondientes a los mismos datos iniciales y de contorno para los sistemas (I), (II), (III).

\section{Dependencia del avance de la frontera libre con la temperatura Tb del baño de aceite.}

Supóngase 2 procesos de freído que ocurren en idénticas condiciones excepto que un caso la temperatura del baño de aceite es $T_{b}^{2}$ y en el otro $T_{b}^{1}$, teniéndose por ejemplo que

$$
T_{b}^{2}>T_{b}^{1}
$$

La condición dada por (49) implica la siguiente desigualdad para el término $\mu \int_{0}^{t} F(\eta) d \eta$ relacionado al flujo de calor F que ingresa hacia la porción de papa desde el baño de aceite durante el freído

$$
\mu \int_{0}^{t} F_{1}(\eta) d \eta<\mu \int_{0}^{t} F_{2}(\eta) d \eta
$$

donde con $\mathrm{F}_{1}$ y $\mathrm{F}_{2}$ se ha denotado a los flujos en correspondencia con $T_{b}^{1}$ y $T_{b}^{2}$ respectivamente. Supongamos que el orden de los datos establecidos por (49) conlleva al siguiente resultado de comparación para los avances de la frontera libre $\sigma(\mathrm{t})$

$$
\sigma_{2}(\mathrm{t})<\sigma_{1}(\mathrm{t})
$$

correspondiendo $\sigma_{2}$ al dato $T_{b}^{2}$ y $\sigma_{1}$ al $T_{b}^{1}$. En vista de (51), ahora se puede escribir:

$$
\begin{aligned}
& \sigma_{1}(t)-\sigma_{2}(t)=\gamma\left[\int_{0}^{\sigma_{1}(t)} U_{1}(Z, t) d Z-\int_{0}^{\sigma_{2}(t)} U_{2}(Z, t) d Z\right]+ \\
& \delta\left[\int_{\sigma_{1}(t)}^{1} T_{1}(Z, t) d Z-\int_{\sigma_{2}(t)}^{1} T_{2}(Z, t) d Z\right]+\mu\left[\int_{0}^{t}\left[F_{1}(\eta)-F_{2}(\eta)\right] d \eta\right]
\end{aligned}
$$


Teniendo presente de que seguirian valiendo las comparaciones establecidas por (44), (45), y en vista de que $\mu>0$ y lo consignado por (50), de (52) se concluye que el segundo miembro resulta negativo, debiendo ser el primer miembro positivo.

Debe entonces darse lo contrario:

$$
T_{b}^{2}>T_{b}^{1} \Rightarrow \sigma_{2}(t) \geq \sigma_{1}(t), \quad t>0
$$

como se espera naturalmente.

Dependencia del avance de la frontera libre con la temperatura inicial de la porción de papa.

Ahora se tienen dos procesos de freído cuyas condiciones operativas difieren únicamente en la temperatura inicial $\mathrm{T}_{0}$ de la porción de papa a freir. Para tomar un caso consideremos

$$
T_{0}^{1}>T_{0}^{2}
$$

Ahora, la desigualdad (55) implica la siguiente para el término relacionado a la condición inicial en (46):

$$
\int_{0}^{1} W_{1}(z) d z<\int_{0}^{1} W_{2}(z) d z
$$

De aquí en más se procede con un argumento similar al aplicado para deducir el resultado expresado por (53) para la dependencia de $\sigma(t)$ con $\mathrm{T}_{\mathrm{b}}$. Así, se obtiene el resultado:

$$
T_{0}^{1}>T_{0}^{2} \Rightarrow \sigma_{1}(t) \geq \sigma_{2}(t), \quad t>0
$$

\section{REFERENCIAS}

[1] FARKAS, B.E., SINGH R.P., \& RUMSEY, T.R. (1996). "Modeling heat and mass transfer in immersion frying, Part I: Model development”, Journal of Food Engineering, 29, 211-226.

[2] VILLA, L.T. (2004). "Freido por inmersión profunda: Modelado y análisis matemático de la dinámica inicial”, Revista FACENA, 20, 47-63. 